\title{
NOVEL SOFTWARE IN MATLAB DESIGNED FOR UWB SENSOR-BASED LOCALIZATION OF PEOPLE IN 2D AND 3D SPACE
}

\author{
Michal ŠVINGÁL*, Dušan KOCUR**, Jana FORTES** \\ *K-Mlab organizational unit of Ilmsens GmbH, University science park TECHNICOM, Němcovej 5, 04001 Košice, \\ E-mail:michal.svingal@ilmsens.com \\ ** Department of Electronics and Multimedia Communications, Faculty of Electrical Engineering and Informatics, Technical \\ University of Košice, Letná 9, 04200 Košice, E-mail: dusan.kocur@tuke.sk, jana.fortes@tuke.sk
}

\begin{abstract}
Detection, localization, and tracking of people in $2 D$ or $3 D$ space using Ultra-wideband (UWB) short-range sensors is a subject of worldwide intensive research. In this paper, we describe a proposal for a novel software called UWB-PerLoc-2D3D. This software has been created in a MATLAB programming environment and designed to process UWB radar signals for the purpose of movement monitoring of human targets in $2 D$ and $3 D$ space. We present an evaluation of the proposed software properties as well as examples of its utilization in practice.
\end{abstract}

Keywords: people localization, software in MATLAB programming environment, radar signal processing, UWB sensor

\section{INTRODUCTION}

When dealing with emergencies where human lives may be endangered, a range of practical applications for people localization using Ultra-wideband (UWB) short-range radars can be employed. Their usage is advantageous mainly in cases where it is not possible to use standard facilities intended for searching for people in danger. UWB sensors operating in the properly chosen frequency band allow to detect and localize people not only in situations with direct visibility, but also persons situated behind a nonmetallic obstacle, e.g. a wall, or in the environment with a very low optical visibility, e.g. in smoke, dust, darkness, dense fog or through foliage [1].

Nowadays, it is frequently discussed the topic of life quality assurance for elderly people. Many family members cannot provide senior care for various reasons. It often happens that an older person does not wish to be monitored by a camera system. The possibility of assisted living with UWB sensors is one of the alternatives for detecting their non-standard behavior. UWB radar system is used to monitor the vital functions of people and changes in their physical position, e.g. detection of an atypical position. The area of processing of signals measured by the UWB sensor is therefore a subject of continuous and intensive research [2].

At the Department of Electronics and Multimedia Telecommunications (KEMT) of the Technical University in Koice (TUKE), ongoing research in the field of UWB sensor systems is performed as well [3]. One part of it is focused on the development of new signal processing methods applied for people detection, localization, and tracking with UWB sensors. For that purpose, it is practical to dispose of user-friendly software enabling the measured radar signals to be processed and evaluated.

As part of the diploma thesis [4], a new software called UWB-PerLoc-2D3D was created in the MATLAB programming environment. It is designed for the offline processing of measured UWB radar signals with the aim to localize moving people in $2 \mathrm{D}$ and $3 \mathrm{D}$ space. The UWBPerLoc-MAT program, which was developed within the bachelor's thesis [5], was used as a basis for the design of new software. In contrast to it, UWB-PerLoc-2D3D offers many improvements, which are described in the following sections of this paper. After evaluation of the proposed software properties, we conclude the paper with examples of its practical usage.

\section{UWB-PERLOC-2D3D SOFTWARE DESIGN}

The purpose of the UWB-PerLoc-2D3D software in the MATLAB programming environment is to ensure effective setting of parameters of individual methods of radar signal processing procedure for moving person detection, localization, and tracking (hereinafter SPMP procedure) and subsequently, process signals measured by UWB sensors with the intention to localize moving people in $2 \mathrm{D}$ or $3 \mathrm{D}$ space [6], [7]. It means it represents an offline processing system of UWB radar signals, which thanks to the implementation of advanced association techniques and multiple target tracking (MTT) system enable positioning of several moving people at the same time.

In the design of the program UWB-PerLoc-2D3D, we based on the bachelor's thesis [5], in which UWB-PerLocMAT software was created for the processing of signals obtained from radar system operating only with one transmitting ( $\mathrm{Tx})$ and two receiving antennas $(\mathrm{Rx})$. In contrast to it, the program UWB-PerLoc-2D3D is extended by algorithms for locating people in 3D space [8], [9]. Based on this, it was necessary to adjust a chain that calculates the SPMP so that it estimates the distances of moving people from radar signals measured from four receiving antennas. Also, newly created software versus the previous one has many improvements and new features in terms of software controllability as well as its implementation. The most important ones are briefly described in the following subsections.

\subsection{Design of folder structure for better orientation between individual $\mathrm{m}$-files}

In the original version of the software, all the necessary $\mathrm{m}$-files were in one folder. Such a file structure seems 
impractical on the part of the developer. Therefore, a new structure has been proposed, which ensures that the m-files mediating the calculation of individual SPMP phases are located in separate folders, which ultimately improves the orientation between software algorithms [4].

\subsection{Using the concept of nested functions for programming of the software main function}

As the proposed software has a graphical user interface (GUI), this interface had to be defined as part of the software development. In the UWB-PerLoc-MAT program, the GUIDE tool was used for this purpose, the advantages of which are described in detail in [5]. However, it turned out that for the design of some other functions of the software it is more suitable to have a graphical interface defined programmatically using the functions of MATLAB. Therefore, when programming the main function of the software, the concept of nested functions [10] was chosen, the use of which brought the following benefits:

- elimination of global variables,

- possibility to set the way of displaying individual interactive components when changing the size of the main application window,

- the ability to implement a toolbar to change the way graphical outputs are displayed.

The final graphical interface of the UWB-PerLoc-2D3D program is shown in Fig. 1

\subsection{Possibility of reading data of various formats}

Former UWB-PerLoc-MAT software is unclear about the type of data to be read. This ambiguity arises from the fact that when selecting the type of data to be read by the software, the options "Radar 1", "Radar 2" and "Radar 3" are available according to the UWB sensor with which the measurement was performed. A much better solution for selecting the data type is to specify the types of real-time software with which the measurement was performed and the corresponding output data format. Additionally, the selection of the following types of partially processed data was implemented: pre-processed radar data, data with subtracted background, filtered data, normalized data, detector output, and output of the suppression of the false targets.

\subsection{Implementation of all available SPMP algorithms}

One of the biggest disadvantages of the original software is the number of implemented SPMP methods. The source of software implementations of these methods was the software Toolbox_UWB, which was gradually designed in TUKE during the UWB project solving and is used to process radar signals to locate moving people in $2 \mathrm{D}$ space. This software is described in more detail in [5]. To maximize the efficiency of person localization, only the algorithms that provide the best results have been implemented in UWB-PerLoc-MAT. However, when developing new algorithms, it is appropriate to have other methods to analyze their mutual functionality for subsequent research. That is the reason why all available SPMP algorithms have been implemented in UWB-PerLoc-2D3D, of which e.g. the wall effect compensation algorithm can be mentioned. The absence of this algorithm in the original software caused significant localization inaccuracies when data from wall measurements were processed. Therefore, the newly created software also includes the option of setting wall parameters [11]. Overview of other SPMP methods implemented in UWB-PerLoc-2D3D is listed in Table 1 .

Table 1 SPMP phases and methods implemented in UWB-PerLoc-2D3D

\begin{tabular}{|ll|}
\hline SPMP phases & SPMP methods \\
\hline Background subtraction & $\begin{array}{l}\text { Simple averaging, Windowed averaging, Exponential averaging, } \\
\text { Adaptive exponential averaging, Gaussian averaging, Gaussian mixture, } \\
\text { Adaptive gaussian }\end{array}$ \\
\hline Range filtering & Passband filtering, Non-linear filtering \\
\hline Normalization & Column normalization, Adaptive normalization, Weighted normalization \\
& Automatic gain control, Advanced normalization \\
\hline Detection & CFAR detector, (N,k) detector, IPCP detector \\
\hline False alarm supression & Logic detector, Median filtering \\
\hline TOA estimation & $\begin{array}{l}\text { Smoothing 1D, 2D Trace method, Trace estimation, Trace online, } \\
\text { Trace connection }\end{array}$ \\
\hline Localization & $\begin{array}{l}\text { Direct calculation, New MT localization, Optimal conditions method, } \\
\text { Newton iteration method }\end{array}$ \\
\hline Tracking & $\begin{array}{l}\text { Median tracking filter, Least-square tracking, Linear Kalman filter, } \\
\text { MTT 2D, MTT 3D }\end{array}$ \\
\hline
\end{tabular}




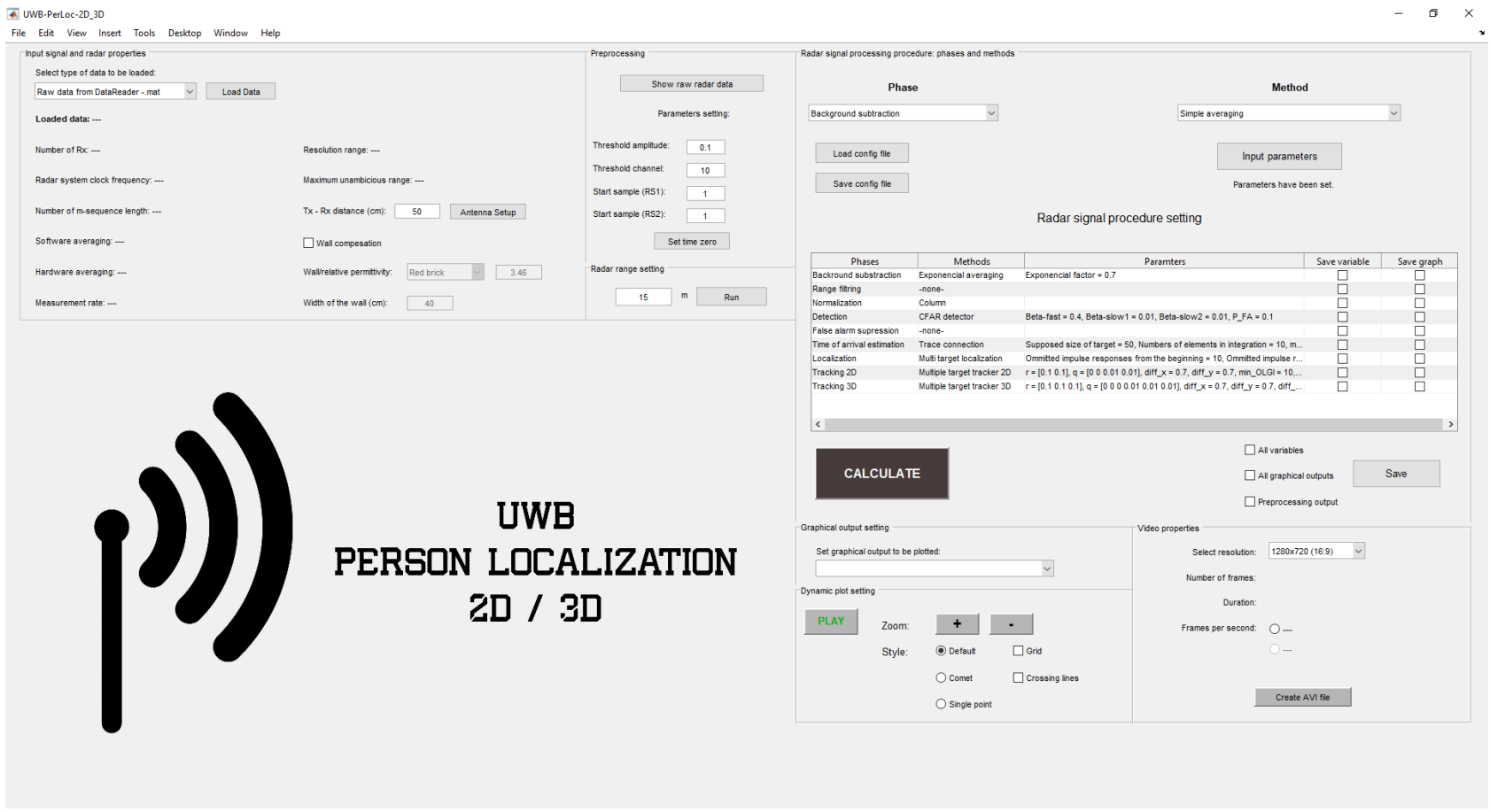

Fig. 1 UWB-PerLoc-2D3D graphical interface

\subsection{More efficient realization of the program implementation of the main chain operations}

One of the tasks of the diploma thesis [4] was to create a toolbox in the MATLAB programming environment, which contains the UWB-PerLoc-2D3D program itself, as well as individual algorithms of signal processing phases for the possibility of creating new methods enabling the SPMP calculation. During the preparation of these algorithms, the $U W B_{-}$processing function was created, which can be effectively used to program the entire chain of operations used to calculate the SPMP. This procedure was also applied in the UWB-PerLoc-2D3D software. Compared to the original software, the time-consuming nature of the SPMP calculation is much lower, as the program during execution of calculation of individual methods does not display each graphical output of the chain of operations, but displays only the output of the last selected method.

As the UWB-PerLoc-2D3D software has a graphical interface, the user triggers various functions of the UWBPerLoc-2D3D program by interacting with the individual components of the interface. More specifically, for example, the input data is read, various SPMP methods and parameters are set, as well as the SPMP calculation itself is performed. Therefore, it is desirable to have the operations of setting the parameters and performing the main calculation separated, as can be seen in Fig. 2

\subsection{Correction of the process of graphical output saving}

When saving graphical outputs, in the original software version, it was necessary to display each graphical output in a separate window before the program saved this output.

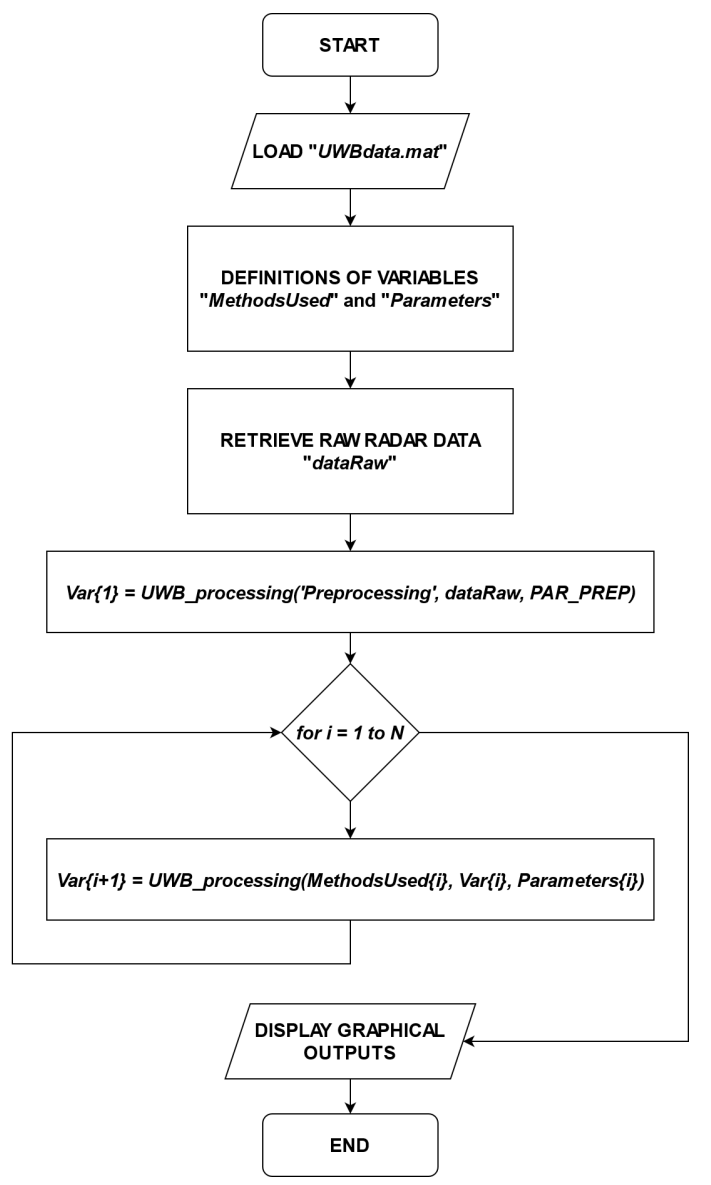

Fig. 2 Utilization of the $U W B_{-}$processing function in UWB-PerLoc-2D3D main chain (flowchart of example2)

After saving, the displayed window was closed. For this reason, storing a large number of graphical outputs 
was very impractical, and from the user's point of view, it acted as a software error. This imperfection has been suppressed in the new version. There is also added option to save graphical outputs in .jpg, .png, .fig and .avi formats in the UWB-PerLoc-2D3D software.

\section{UWB-PERLOC-2D3D SOFTWARE FEATURES}

The most significant improvement of the UWB-PerLoc2D3D software compared to the original UWB-PerLocMAT software, in addition to the above-described improvements in terms of software control and implementation, is the ability to process UWB radar signals obtained from a multistatic radar operating with $1 \mathrm{Tx}$ and $4 \mathrm{Rx}$, and thus to localize moving persons in $3 \mathrm{D}$ space. This property was ensured by the implementation of new localization algorithms, namely the 3D-2D method, the approximation of the 3D-2D method, the optimization method, and the Newton iteration method [7], [8], [9]. Because these algorithms were not given sufficient attention from the point of view of the correctness of their program implementation, the analysis of their properties was performed on a sample of synthetic data using computer simulation (Fig. 3).

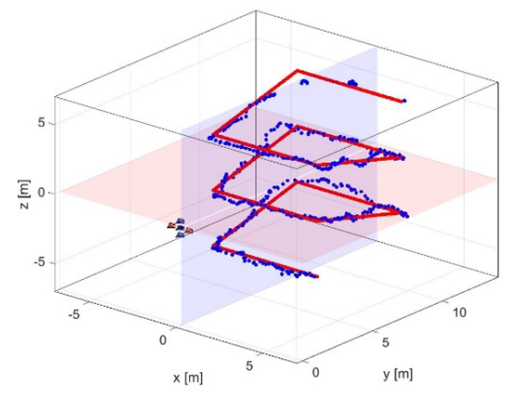

Fig. 3 Graphical output of the tracking phase applied to the synthetic data

Due to the preparation of functions ensuring the calculation of the mentioned algorithms and functions for displaying the estimated coordinates of targets in the 3D coordinate system, it proved appropriate to create this simulation in the MATLAB programming environment. The main benefit of such a simulation was the preparation of various other functions for the calculation of not only above mentioned algorithms but also many others, which were created as a combination of available algorithms for locating and tracking targets in 2D and 3D space. As the designed simulation is based on testing localization algorithms on a sample of synthetic data, it was possible to analyze these algorithms in terms of localization accuracy [4]. The mentioned procedure greatly facilitated the design of the UWB-PerLoc2D3D program, and therefore it can be stated that this process was suitable for solving the task of implementation of the given algorithms into the newly created software.

As part of the diploma thesis [4], the UWB Toolbox was designed in the MATLAB programming environment, too. Its file structure is designed to guide future developers to use the helping functions $U W B_{-}$processing, $U W B_{\text {_imagesc, }} U W B_{-}$plot $2 D$, and $U W B_{-}$plot $3 D$. These functions can be easily used to create other possible software products for the development of UWB sensor applications. During the development of toolbox functions, the basic concept of the SPMP calculation was defined, which was used not only in the UWB-PerLoc-2D3D program itself but also in simple programs created to demonstrate its usage for future developers. The flowchart of one of them, named example2, is shown in Fig. 2 .

To verify the properties of the UWB-PerLoc-2D3D software, an experimental measurement with a multistatic UWB radar was performed within the diploma thesis [4] and subsequently, measured radar signals were processed using the proposed program. Based on the obtained experimental results, it has been affirmed that the software is able to correctly localize moving persons in $3 \mathrm{D}$ space (Fig. 4). In addition to calculating the entire radar signal processing procedure, the software has some other positive features, including the dynamic rendering of graphical outputs, the ability to read data obtained by one of the two types of real-time measuring software, or processed data from some phases of the procedure, storing graphical outputs of the program in .jpg, .png, .fig formats, or even saving the video in avi format.

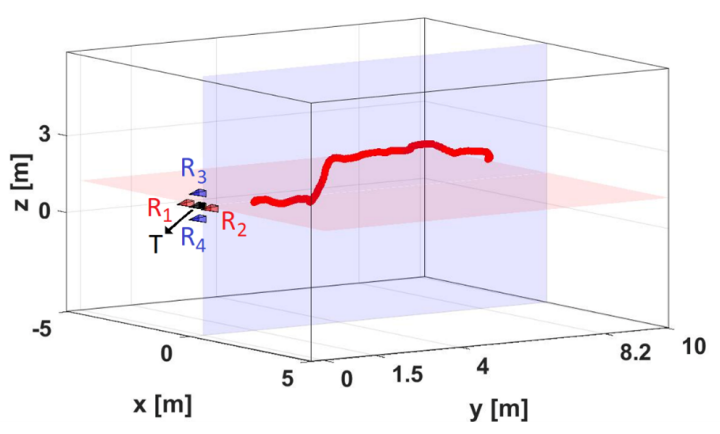

Fig. 4 Graphical output of the tracking phase applied to the measured data.

Of course, the proposed software is also characterized by negative properties. In the case of UWB-PerLoc-2D3D, its biggest disadvantage is the high memory requirements. This program is designed in the way that all variables containing the processed signals are still available in memory after the calculation of the procedure for the purpose of their display or any other operation. This feature could be eliminated by using a different programming approach in which the program works with storage, or when calculating a given variable, the program stores this variable in a predefined place in the storage, respectively. Subsequently, when an operation is requested, for example, the display of graphical output, this variable is loaded into memory. In the case of the $U W B_{-}$processing function of the UWB Toolbox, its negative feature is a relatively complex process of defining input parameters and also accessing output parameters, as the inputs and outputs of this function are defined as cell fields [12]. This complexity results from the fact that when designing this function, the aim was to create a concept according to which the whole UWB radar signal processing procedure can be easily programmed. 


\section{CONCLUSIONS}

From the way of working with the program UWBPerLoc-2D3D, which is simple and intuitive thanks to the graphical interface, it can be stated that this software can be used in educational and scientific research activities at TUKE. Its pilot testing took place within the subject UWB sensor network in the winter semester of the school year 2020/2021. UWB-PerLoc-2D3D software is also actively used in ongoing research and development in the field of people monitoring performed at KEMT.

Given that in many cases it appears practical to have software that can detect, localize and track moving persons in real-time, the idea for extending this work is to implement such functionality in the software UWB-PerLoc2D3D. Another extension can be related to the addition of signal processing methods for detection, localization, and tracking of static people based on their vital signs and in such a way enable to track people changing their motion activity, too.

\section{ACKNOWLEDGEMENT}

This work was supported by the Slovak Research and Development Agency under contract No. APVV-18-0373 and by the Scientific Grant Agency (VEGA) under contract No. $1 / 0584 / 20$.

\section{REFERENCES}

[1] FONTANA, R. J.: Recent Applications of Ultra Wideband Radar and Communications Systems. Book chapter in Ultra-Wideband, Short-Pulse Electromagnetics 5, Isbn: 978-0-306-47948-9, 2002, pp. 225234. http://dx.doi.org/10.1007/0-306-47948-6_ 27

[2] ALARIFI, A. - AL-SALMAN, A. - ALSALEH, M. - ALNAFESSAH, A. - ALHADHRAMI, S. - ALAMMAR, M. A. - AL-KHALIFA, H. S.: Ultra wideband indoor positioning technologies: Analysis and recent advances, Sensors (Basel) 16, No. 5 (May 2016) 1-36

[3] KOCUR, D. et al.: Detekcia, lokalizácia a sledovanie osb UWB radarmi krtkeho dosahu. TUKE, Koice, 2016. ISBN 987-80-553-2648-1

[4] ŠVINGÁL, M.: UWB-PerLoc-2D3D: Softvr uren na vvoj aplikci UWB senzorov na lokalizciu osb v 2D a 3D priestore. Diplomov prca, TUKE, Koice, 2019.

[5] ŠVINGÁL, M.: UWB-PerLoc-MAT: Softvr v programovacom prostred MATLAB uren na vvoj aplikci UWB senzorov na lokalizciu osb. Bakalrska prca, TUKE, Koice, 2017.

[6] ROVŇÁKOVÁ, J.: Complete signal processing for through wall target tracking by $\mathrm{M}$-sequence UWB radar system. Dizertan prca, TUKE, Koice, 2009.

[7] ŠVECOVÁ, M.: Lokalizcia cieľa UWB radarovm systmom. Dizertan prca, TUKE, Koice, 2009.

[8] ŠVECOVÁ, M. - KOCUR, D. - DEMČÁK, J. GAMCOVÁ, M. - SLOVÁK, S.: Through-the-floor localization of a static person by multistatic $U W B$ radar, Microwave and Optical Technology Letters 61, No. 3 (2019) 825-831 http://dx.doi.org/10.1002/ mop.31609

[9] BUŠA, J.: Target Localization by UWB Signals, In: Dimov I., Fidanova S., Lirkov I. (eds) Numerical Methods and Applications, Lecture Notes in Computer Science, Springer 8962, No. 1 (2015) 254-261

[10] MathWorks: Nested Functions. [online]. The MathWorks, Inc. [cited 29.6.2021]. Available on the internet https://www.mathworks.com/help/matlab/ matlab_prog/nested-functions.html

[11] ROVŇÁKOVÁ, J. - KOCUR, D.: Compensation of wall effect for through wall tracking of moving targets, In: Radioengineering, Part 2, Special Issue on Workshop of the COST Action IC0803: $R F$ /Microwave Communication Subsystems for Emerging Wireless Technologies (RFCSET) 18, No. 2 (2009) 189-195 ISSN 1210-2512

[12] MathWorks: Cell Arrays. [online]. The MathWorks, Inc. [cited 29.6.2021]. Available on the internet https://www.mathworks.com/help/matlab/ cell-arrays.html

Received June 30, 2021, accepted September 27, 2021

\section{BIOGRAPHIES}

Michal Švingál was born in 1996 in Lučenec, Slovakia. He received his Ing. (M.Sc.) degree in Telecommunications from the Faculty of Electrical Engineering and Informatics, Technical University of Košice, in 2019. He is currently working at the K-Mlab organization unit of Ilmsens $\mathrm{GmbH}$. His research is focused on UWB sensor networks to be applied for the detection, localization, and tracking of moving and motionless persons.

Dušan Kocur was born in 1961 in Košice, Slovakia. He received his Ing. (M.Sc.) and CSc. (Ph.D.) degrees in Radioelectronics from the Faculty of Electrical Engineering, Technical University of Košice, in 1985 and 1990, respectively. Now, he is the full professor at the Department of Electronics and Multimedia Communications of his Alma Mater. His research interests are radar signal processing, UWB technologies, and their applications.

Jana Fortes was born in 1983 in Michalovce, Slovakia. She received her M.Sc. degree in Mathematics from the Faculty of Science, Pavol Jozef Šafárik University in Košice and her Ph.D. degree in Electronics from the Faculty of Electrical Engineering, Technical University of Košice, in 2006 and 2009, respectively. From 2009 to 2014 and since 2020, she has been a researcher at the Department of Electronics and Multimedia Communications of Technical University of Košice, Slovakia. Her research interests are in UWB radar signal processing applied for the detection, localization, and tracking of moving or static persons. 